\title{
Emotional Well-Being and Use of Technology During COVID-19 Social Isolation in a Predominantly Minority Older-Adult Population
}

\author{
Sophie Y. Lin ${ }^{1}$ Anuj S. Marathe ${ }^{1}$ Jennifer L. Bryan ${ }^{1,2,3}$. Ali Abbas Asghar-Ali ${ }^{1,2,3}$
}

Received: 4 May 2021 / Revised: 13 October 2021 / Accepted: 1 November 2021 / Published online: 17 November 2021

This is a U.S. government work and not under copyright protection in the U.S.; foreign copyright protection may apply 2021

\section{Introduction}

During the coronavirus disease 2019 (COVID-19) pandemic, stay-at-home orders were issued across most of the USA to mitigate the spread of severe acute respiratory distress syndrome coronavirus 2 (SARS-CoV-2) and prevent over-burdening health systems. The Centers for Disease Control and Prevention (CDC) has reported that older adults and people who have severe underlying medical conditions are at a higher risk of developing more serious complications from COVID-19, and to date, 8 out of 10 deaths reported in the USA have been in adults 65 years or older (CDC, 2020).

Although self-isolation can help decrease transmission and mortality risk, it can lead to social isolation, which has been identified as a "serious public health concern" in older adults because of their increased risk for physical and mental health consequences (Armitage \& Nellums, 2020). Social disconnection from day center venues, community centers, and places of worship during this time can place older adults at greater risk of depression and anxiety (Armitage $\&$ Nellums, 2020). As social isolation increases with age, older adults tend to be more prone to loneliness (Seyfzadeh et al., 2019).

While online technology platforms can be used to stay connected, barriers such as lack of support and low technology literacy (Vaportzis et al., 2017) limit older adults' adoption of new technologies. However, older adults' use of technology has been increasing; as only $14 \%$ of those 65 and older reported using the internet compared to $67 \%$ in 2017

Ali Abbas Asghar-Ali

asgharal@bcm.edu

1 Baylor College of Medicine, Houston, TX, USA

2 Houston VA HSR\&D Center for Innovations in Quality, Effectiveness and Safety, Michael E. DeBakey VA Mecical Center, Houston, TX, USA

3 VA South Central Mental Illness Research, Education and Clinical Center, Houston, TX, USA
(Anderson \& Perrin, 2017) Additionally, social media usage has increased among those 65 and older, from $27 \%$ in 2013 to $34 \%$ in 2017 (Anderson \& Perrin, 2017). Barriers to technology use are reported by adults aged 65 and older, including feeling less confident in their ability to use technology and being more likely to need help learning a technological device than individuals younger than 65 (Anderson \& Perrin, 2017). Increased isolation during COVID-19 may compel older adults to engage with technology to maintain their social networks and a sense of belonging. To our knowledge, no study has yet investigated predominantly minority older adults' use of technology platforms during COVID-19.

The purpose of our study was to understand how the stayat-home order has affected older adults' activities, use of technology platforms, and sense of loneliness.

\section{Methods}

This cross-sectional study of older adults from BakerRipley, a collection of community senior centers in Houston, Texas, was approved by the Baylor College of Medicine Institutional Review Board. Contact information for 113 members was provided by BakerRipley, and phone interviews were conducted between April 20, 2020 and May 1, 2020. Of the 113 older adults contacted, 60 did not answer the phone or did not want to participate. Participants were not compensated for their time, and there were no incentives to participate. There was no consent for this study. Houston's stay-at-home-order had been in effect since March 24, 2020. All participants who started the survey completed it.

The survey started with demographic questions (e.g., age, living situation, internet access). Next, participants were asked about how their activities have changed since the stay-at-home order (e.g., grocery shopping, meeting friends) on a scale from 1 (completely stopped) to 6 (much more often) with an option to say that activity was not applicable to their life before the stay-at-home order. Next, we asked 
the six questions about frequency of messaging, video calling, and social media use before and then during the stay-at home-order on a scale from 1 (never) to 4 (daily). The University of California at Los Angeles Three-Item Loneliness Scale (Hughes et al., 2004) measured perceived loneliness ( $\alpha=0.84$ ) on a scale from 1 (hardly ever) to 3 (often). Participants were asked open-ended questions. Open-ended questions asked about interest in learning more about technology and what has made them happy in the past as well as during the stay-at-home order, how they spent their time before and during the stay-at-home order, and how they could be better supported. Prior to conducting the interviews, the survey was approved by BakerRipley stakeholders. The results of the survey were reported back to BakerRipley so that it could better meet the needs of its members.

Data analysis was performed using IBM SPSS version 26 (SPSS Inc., Chicago, IL, USA). Statistical significance between 2 time points was determined with paired sample $t$-tests. Statistical significance among groups of 3 was determined using Welch's ANOVA test because groups were not homogeneous, as determined by Levene's test. To identify the specific areas of group differences, Games-Howell posthoc analysis was performed. $P$ values of $<0.05$ were considered statistically significant. Open-ended questions were analyzed with reflexive thematic analysis (Braun \& Clarke, 2019).

\section{Results}

A total of 53 older adults were included in the analysis.

\section{Quantitative Analysis}

Our respondents were $81.1 \%$ (43/53) female, $67.9 \%$ (36/53) African American, 18.9\% Hispanic (10/53), 11.3\% White (6/53), and $1.9 \%$ (1/53) Mixed. Three age groups were represented: $61-70$ years $(45.3 \%, 24 / 53), 71-80$ years $(34 \%$, $18 / 53)$, and $80+$ years $(20.8 \%, 11 / 53)$. In terms of living situation, 35.8\% (19/53) lived alone, 28.3\% (15/53) lived with pets or had a caregiver, and $35.8 \%$ (19/53) lived with family members. All of our respondents were following the stayat-home order, and $98.1 \%$ (52/53) agreed with social distancing. Self-reported changes in frequency of older adults' activities during the stay-at-home order compared to before the stay-at-home order can be found in Table 1. The entire sample stopped eating out at restaurants, and all but one reported stopping attending religious services. Participants reported that they had completely stopped to much less often visiting friends and taking public transportation. Participants reported grocery shopping and visiting family much less often to less often as compared to before the pandemic.
Table 1 Self-reported changes in the frequency of older adults' activities before the stay-at-home order compared to during the stay-athome order

\begin{tabular}{llll}
\hline & $\boldsymbol{N}$ & $\boldsymbol{M} \pm \boldsymbol{S \boldsymbol { D } ^ { \mathrm { a } }}$ & Range \\
\hline Groceries & 48 & $2.42 \pm 1.09$ & $(1.00,4.00)$ \\
Friends & 48 & $1.54 \pm 0.74$ & $(1.00,3.00)$ \\
Family & 50 & $2.74 \pm 1.21$ & $(1.00,6.00)$ \\
Religious service & 40 & $1.08 \pm 0.47$ & $(1.00,4.00)$ \\
Public transport & 19 & $1.21 \pm 0.63$ & $(1.00,3.00)$ \\
Restaurant & 47 & $1.00 \pm 0.00$ & $(1.00,1.00)$ \\
\hline
\end{tabular}

${ }^{\mathrm{a}} 1=$ completely stopped, $2=$ much less often, $3=$ less often, $4=$ about the same, $5=$ more often, $6=$ much more often

Most of our respondents $(79.2 \%, 42 / 53)$ had internet access. Group differences were tested from before the pandemic to during the stay-at-home order with a paired sample $t$-test. During the pandemic, older adults showed statistically significant increases in messaging $(t(52)=-3.04$, $p=0.004)$, video calling $(t(52)=-4.81, p<0.001)$, and social media use $(t(52)=-2.84, p=0.006)$. Three Welch's ANOVA were used to see if there were group differences for messaging, video calling, and social media usage during the stay-at-home order. The analysis revealed significant differences between age groups and messaging (Welch's $F(2$, $26.25)=130.46, p<0.001)$, video calling (Welch's $F(2$, $30.58)=5.04, p=0.01)$, and social media use (Welch's $F(2$, $30.30)=9.91, p<0.001)$. Games-Howell post hoc analyses were used to determine where the group differences were. The largest group differences were found between the 61-70 and $80+$ age groups, with the $61-70$ age group using each technology platform significantly more than the $80+$ age group $(p<0.05)$. In addition, 58.5\% (31/53) of our respondents were interested in learning more about different technology platforms.

The mean and standard deviation on the University of California at Los Angeles Three-Item Loneliness Scale were $4.79 \pm 1.96$. Interestingly, there were no statistically significant relationships between age group, living situation, or frequency of technology use and perceived loneliness.

\section{Thematic Analysis}

Older Adults with Frequent Family Contact Feel Supported Respondents who lived with family members or had family members who visited them reported, "I got a lot of support! I talk to my friends and family on the phone, and they always check on me!" Some also learned how to use technology platforms from their children or grandchildren. In contrast, older adults who lived alone with no immediate family support or who had physical limitations voiced greater challenges, especially with groceries. One respondent who is wheelchair-bound shared, “...my grandchildren 
were the ones who usually helped me [with household tasks], but I don't see them now." Another respondent who lived alone expressed, "I have been actively seeking a counselor, but I have been struggling to find the right person."

Older Adults Find Happiness in Spirituality When respondents were asked, "Currently, what brings you happiness?" many responded "reading, especially the Bible," "God," or "church." One lady, "... [texted] prayers or pictures to other people to bring some joy or happiness in their lives."

Older Adults Derive Social Support from Community Venues All of our respondents participated in activities at BakerRipley. When asked what brought them happiness before the pandemic, many people mentioned "BakerRipley." However, they described their current activities as more "limited" and "boring" because they "can't go out." As one respondent shared in depth: "I used to go to the senior center five times a week. We would go on field trips, line dance, and go to the theater. I would see my friends and talk to people my age at the center."

Self and Socially Isolated Older Adults Do Not Necessarily Feel Lonely Despite having to stay at home, older adults did not report increased loneliness. Many of our respondents, especially women, remained connected with their friends by "talking to [them] on the telephone" every day. Others kept themselves busy with "reading," "writing," "TV," or "walking outside."

\section{Discussion}

Before the pandemic, all of our respondents participated in activities at BakerRipley and primarily socialized with their friends in-person. Our study revealed that, during the stay-at-home order, older adults were able to overcome isolation and maintain their social connections by engaging more with technology. Video calling platforms showed the greatest increase in use, which may be explained by church activities transitioning to Zoom and older adults' appreciation for face-to-face communication. Those in the oldest age group $(80+$ years $)$ showed the smallest increase in technology use, which highlights a potential digital divide within older adults themselves due to differences in technology exposure, increased age-related cognitive and physical changes, or a perceived inability to learn. However, in general, respondents had positive attitudes toward technology and expressed interest in technology classes. Among those who were not interested, many cited having family members available to help them or believed that they were "too old to learn." Since technology can enhance interpersonal relationships (Chopik, 2016), community venues should consider creating instructional materials or hosting educational sessions to teach older adults more about common technology platforms.

We found no statistically significant relationships between living situation or frequency of technology use and perceived loneliness. Our results may be explained by notable differences between living alone and feeling lonely (Perissinotto et al., 2012; Yanguas et al., 2018; BedardThomas et al., 2019). Loneliness tends to manifest when there is a perceived lack of social engagement (BedardThomas et al., 2019); however, our analyses suggest that our respondents, even those who lived alone, hardly ever felt lonely because they were still able to remain connected with their friends and families. Additionally, studies have shown that group interventions focused on strengthening social bonds and friendship are the most effective in alleviating loneliness experienced by older adults (Yanguas et al., 2018). Given that our respondents had been actively engaged with BakerRipley, they may have had stronger social bonds than older adults who had not been attending a senior center. Men and women also maintain social networks in different ways, with women spending about twice as much time on the telephone as men (Szell \& Thurner, 2013). Since most of our respondents were women, we believe that they were more likely to derive support from their existing groups via telephone check-ins than their male counterparts.

A limitation of our study is that all of our respondents are members of a community center that provides support and resources. Our results may not be generalizable to older adults who are not part of a similar community. The study used self-report measures, and there was no comparison group. It is possible that our population under- or overreported activities during the stay-at-home order. Additionally, our study was completed during the first few weeks of the stay-at-home order; results may have changed if the survey had been completed at different time points during the pandemic.

Our study suggests that older adults are adapting to unprecedented challenges during the initial stage of the pandemic. However, some interventions could be implemented to better support them, such as distributing pamphlets with key resources, developing a more robust grocery delivery service, and offering instructional technology classes. Additional research is needed to elucidate how the needs of older adults have changed throughout the pandemic.

Author Contribution All authors had a role in study concept and design, acquisition of subjects and/or data, analysis and interpretation of data, and preparation and final approval of manuscript.

Funding This study was partially supported by the use of facilities and resources at the Houston VA HSR\&D Center for Innovations in 
Quality, Effectiveness and Safety (CIN13-413) and the VA South Central Mental Illness Research, Education and Clinical Center.

\section{Declarations}

Disclaimer The opinions expressed reflect those of the authors and not necessarily those of the Department of Veterans Affairs, the US government, or Baylor College of Medicine.

Conflict of Interest The authors declare no competing interests.

\section{References}

Anderson, M., \& Perrin, A. (2017). Technology adoption climbs among older adults. Pew Research Center, Washington, D.C. Last accessed 13 Oct 2021. Available at https://www.pewresearch.org/ internet/2017/05/17/barriers-to-adoption-and-attitudes-towardstechnology/

Armitage, R., \& Nellums, L. B. (2020). COVID-19 and the consequences of isolating the elderly. The Lancet Public Health, 5(5), e256. https://doi.org/10.1016/S2468-2667(20)30061-X

Bedard-Thomas, J., Gausvik, C., Wessels, J., Regan, S., Goodnow, K., \& Goroncy, A. (2019). I live alone but don't feel alone: Social isolation and loneliness from the patient perspective. Journal of Patient-Centered Research and Reviews, 6(4), 262-266. https:// doi.org/10.17294/2330-0698.1715

Braun, V., \& Clarke, V. (2019). Reflecting on reflexive thematic analysis. Qualitative Research in Sport, Exercise and Health, 11(4), 589-597.
CDC. (2020, February 11). Coronavirus Disease 2019 (COVID-19). Centers for Disease Control and Prevention. https://www.cdc.gov/ coronavirus/2019-ncov/need-extra-precautions/older-adults.html

Chopik, W. J. (2016). The benefits of social technology use among older adults are mediated by reduced loneliness. Cyberpsychology, Behavior, and Social Networking, 19(9), 551-556. https://doi.org/ 10.1089/cyber.2016.0151

Hughes, M. E., Waite, L. J., Hawkley, L. C., \& Cacioppo, J. T. (2004). A short scale for measuring loneliness in large surveys: Results from two population-based studies. Research on Aging, 26(6), 655-672. https://doi.org/10.1177/0164027504268574

Perissinotto, C. M., Cenzer, I. S., \& Covinsky, K. E. (2012). Loneliness in older persons: A predictor of functional decline and death. Archives of Internal Medicine, 172(14), 1078-1083. https://doi. org/10.1001/archinternmed.2012.1993

Seyfzadeh, A., Haghighatian, M., \& Mohajerani, A. (2019). Social isolation in the elderly: The neglected issue. Iranian Journal of Public Health, 48(2), 365-366.

Szell, M., \& Thurner, S. (2013). How women organize social networks different from men. Scientific Reports, 3(1), 1214. https://doi.org/ 10.1038/srep01214

Vaportzis, E., Giatsi Clausen, M., \& Gow, A. J. (2017) Older adults' perceptions of technology and barriers to interacting with tablet computers: A focus group study. Frontiers in Psychology 8. https://doi.org/10.3389/fpsyg.2017.01687

Yanguas, J., Pinazo-Henandis, S., \& Tarazona-Santabalbina, F. J. (2018). The complexity of loneliness. Acta Bio Medica : Atenei Parmensis, 89(2), 302-314. https://doi.org/10.23750/abm.v89i2. 7404

Publisher's Note Springer Nature remains neutral with regard to jurisdictional claims in published maps and institutional affiliations. 